\title{
Thermodynamics of Anharmonic Systems: Uncoupled Mode Approximations for Molecules
}

\author{
Yi-Pei Li ${ }^{l}$,Alexis T. Bell ${ }^{1,3}$, and Martin Head-Gordon ${ }^{* 2,3}$ \\ ${ }^{1}$ Department of Chemical and Biomolecular Engineering, University of California, \\ Berkeley, California 94720-1462 \\ ${ }^{2}$ Department of Chemistry, University of California, \\ Berkeley, California 94720-1462 \\ ${ }^{3}$ Chemical Sciences Division, Lawrence Berkeley National Laboratory, \\ Berkeley, California 94720
}

\section{Supporting Information}


Text S1. Cubic spline interpolation.

For a set of $\mathrm{N}+1$ points sampled for a mode $\left(E_{0}, E_{1}, \ldots, E_{N}\right)$, the ith piece of the spline is

$$
\begin{aligned}
V_{i}(Q) & =a_{i}{ }^{\prime}+b_{i}{ }^{\prime}\left(\frac{Q-Q_{i-1}}{\Delta Q_{i}}\right)+c_{i}{ }^{\prime}\left(\frac{Q-Q_{i-1}}{\Delta Q_{i}}\right)^{2}+d_{i}{ }^{\prime}\left(\frac{Q-Q_{i-1}}{\Delta Q_{i}}\right)^{3} \\
& =a_{i}+b_{i} Q+c_{i} Q^{2}+d_{i} Q^{3}
\end{aligned}
$$

where $\Delta Q_{i}=Q_{i}-Q_{i-1}$. With the boundary conditions $V_{1}^{\prime \prime}\left(Q_{0}\right)=V_{N}{ }^{\prime \prime}\left(Q_{N}\right)=V^{\prime \prime}(0)$, the coefficients $a_{i}, b_{i}, c_{i}$, and $d_{i}$ can be derived as

$$
\begin{gathered}
a_{i}=a_{i}^{\prime}-b_{i}^{\prime}\left(\frac{Q_{i-1}}{\Delta Q_{i}}\right)+c_{i}{ }^{\prime}\left(\frac{Q_{i-1}}{\Delta Q_{i}}\right)^{2}-d_{i}{ }^{\prime}\left(\frac{Q_{i-1}}{\Delta Q_{i}}\right)^{3} \\
b_{i}=b_{i}^{\prime}\left(\frac{1}{\Delta Q_{i}}\right)-2 c_{i}{ }^{\prime}\left(\frac{1}{\Delta Q_{i}}\right)\left(\frac{Q_{i-1}}{\Delta Q_{i}}\right)+3 d_{i}{ }^{\prime}\left(\frac{1}{\Delta Q_{i}}\right)\left(\frac{Q_{i-1}}{\Delta Q_{i}}\right)^{2} \\
c_{i}=c_{i}{ }^{\prime}\left(\frac{1}{\Delta Q_{i}}\right)^{2}-3 d_{i}{ }^{\prime}\left(\frac{1}{\Delta Q_{i}}\right)^{2}\left(\frac{Q_{i-1}}{\Delta Q_{i}}\right) \\
d_{i}=d_{i}{ }^{\prime}\left(\frac{1}{\Delta Q_{i}}\right)^{3}
\end{gathered}
$$

with

$$
\begin{aligned}
& a_{i}^{\prime}=E_{i-1} \\
& b_{i}{ }^{\prime}=D_{i-1} \Delta Q_{i} \\
& c_{i}{ }^{\prime}=3 \Delta E_{i}-\left(2 D_{i-1}+D_{i}\right) \Delta Q_{i} \\
& d_{i}{ }^{\prime}=-2 \Delta E_{i}+\left(D_{i-1}+D_{i}\right) \Delta Q_{i}
\end{aligned}
$$

where $\left(D_{0}, D_{1}, \ldots, D_{N}\right)$ is the solution of the following equation:

$$
\left[\begin{array}{ccccc}
\frac{2}{\Delta Q_{1}} & \frac{1}{\Delta Q_{1}} & & & \\
\frac{1}{\Delta Q_{1}} & 2\left(\frac{1}{\Delta Q_{1}}+\frac{1}{\Delta Q_{2}}\right) & \frac{1}{\Delta Q_{2}} & & \\
\vdots & \ddots & \ddots & \ddots & \ddots \\
& & \frac{1}{\Delta Q_{N-1}} & 2\left(\frac{1}{\Delta Q_{N-1}}+\frac{1}{\Delta Q_{N}}\right) & \frac{1}{\Delta Q_{N}} \\
& & \frac{1}{\Delta Q_{N}} & \frac{2}{\Delta Q_{N}}
\end{array}\right]\left[\begin{array}{c}
D_{0} \\
D_{1} \\
\vdots \\
D_{N-1} \\
D_{N}
\end{array}\right]=\left[\begin{array}{c}
3 \frac{\Delta E_{1}}{\Delta Q_{1}{ }^{2}}-\frac{1}{2} V^{\prime \prime}(0) \\
3\left(\frac{\Delta E_{1}}{\Delta Q_{1}{ }^{2}}+\frac{\Delta E_{2}}{\Delta Q_{2}{ }^{2}}\right) \\
\vdots \\
3\left(\frac{\Delta E_{N-1}}{\Delta Q_{N-1}{ }^{2}}+\frac{\Delta E_{N}}{\Delta Q_{N}{ }^{2}}\right) \\
3 \frac{\Delta E_{N}}{\Delta Q_{N}{ }^{2}}+\frac{1}{2} V^{\prime \prime}(0)
\end{array}\right]
$$


Text S2. Anharmonic Hamiltonian matrix.

Since the PES is represented by a collection of splines, the elements of the Hamiltonian matrix have to be calculated piecewise

$$
H_{m n}=\left\langle\varphi_{m}\left|-\frac{\hbar^{2}}{2} \frac{d^{2}}{d Q^{2}}+V(Q)\right| \varphi_{n}\right\rangle=\left\langle\varphi_{m}\left|-\frac{\hbar^{2}}{2} \frac{d^{2}}{d Q^{2}}\right| \varphi_{n}\right\rangle+\sum_{i} \int_{Q_{i-1}}^{Q_{i}} \varphi_{m} V_{i} \varphi_{n} d Q
$$

Using harmonic oscillator functions as the basis set, $H_{m n}$ can be derived as

$$
\begin{aligned}
H_{m n}=\delta_{m n}( & \left.-\frac{1}{2} P(2 m+1)\right)+\delta_{m(n+2)}\left(\frac{1}{2} P \sqrt{m(m-1)}\right)+\delta_{n(m+2)}\left(\frac{1}{2} P \sqrt{n(n-1)}\right) \\
& +\frac{1}{\sqrt{2^{n+m} n ! m ! \pi}} \sum_{i} \int_{Q_{i-1} / R}^{Q_{i} / R} a_{i} H_{m} H_{n} e^{-x^{2}}+b_{i} R H_{m}\left(\frac{1}{2} H_{n+1}+n H_{n-1}\right) e^{-x^{2}} \\
& +c_{i} R^{2} H_{m}\left(\frac{1}{4} H_{n+2}+\left(n+\frac{1}{2}\right) H_{n}+n(n-1) H_{n-2}\right) e^{-x^{2}} \\
& +d_{i} R^{3} H_{m}\left(\frac{1}{8} H_{n+3}+\frac{3}{4}(n+1) H_{n+1}+\frac{3}{2} n^{2} H_{n-1}+n(n-1)(n-2) H_{n-3}\right) e^{-x^{2}} d x
\end{aligned}
$$

with

$$
\begin{gathered}
\int H_{m} H_{n} e^{-x^{2}} d x=\frac{1}{m-n} e^{-x^{2}}\left(n H_{m} H_{n-1}-m H_{n} H_{m-1}\right) \\
\int H_{n} H_{n} e^{-x^{2}} d x=2^{n} n ! \frac{\sqrt{\pi}}{2} \operatorname{erf}(x)-\sum_{i=0}^{n-1} 2^{n-1-i} H_{i+1} H_{i} e^{-x^{2}} \frac{n !}{(i+1) !}
\end{gathered}
$$

where $P=-\frac{\hbar \omega_{j}}{2} ; R=\sqrt{\frac{\hbar}{\omega_{j}}} ; x=\frac{1}{R} Q$. On the other hand, using Fourier series as the basis set, $H_{m n}$ can be derived as

$$
H_{m n}=\delta_{m n}\left(\frac{\left(k_{m} \hbar\right)^{2}}{2}\right)+\sum_{i} V_{m n, i}
$$

where $V_{m n, i}=\int_{Q_{i-1}}^{Q_{i}} \varphi_{m} V_{i} \varphi_{n} d Q ; k_{m}=\frac{m \pi}{L}$. The formula of $V_{m n, i}$ is determined by the basis functions $\varphi_{m}$ and $\varphi_{n}$. For the cases where $m \neq n$, if $\varphi_{m}=\frac{1}{\sqrt{2 L}}$ and $\varphi_{n}=\frac{1}{\sqrt{L}} \sin \left(k_{n} Q\right)$,

$$
\begin{gathered}
V_{m n, i}= \\
\frac{1}{\sqrt{2} L}\left(a_{i} \frac{-\cos \left(k_{n} Q\right)}{k_{n}}+b_{i} \frac{\left(-k_{n} Q\right) \cos \left(k_{n} Q\right)+\sin \left(k_{n} Q\right)}{k_{n}{ }^{2}}+c_{i} \frac{\left(2-k_{n}{ }^{2} x^{2}\right) \cos \left(k_{n} Q\right)+2 k_{n} Q \sin \left(k_{n} Q\right)}{k_{n}{ }^{3}}+\right. \\
\left.d_{i} \frac{-k_{n} Q\left(k_{n}{ }^{2} x^{2}-6\right) \cos \left(k_{n} Q\right)+3\left(k_{n}{ }^{2} x^{2}-2\right) \sin \left(k_{n} Q\right)}{k_{n}{ }^{4}}\right)
\end{gathered}
$$

if $\varphi_{m}=\frac{1}{\sqrt{2 L}}$ and $\varphi_{n}=\frac{1}{\sqrt{L}} \cos \left(k_{n} Q\right)$ 


$$
\begin{gathered}
V_{m n, i}= \\
\frac{1}{\sqrt{2} L}\left(a_{i} \frac{\sin \left(k_{n} Q\right)}{k_{n}}+b_{i} \frac{\left(k_{n} Q\right) \sin \left(k_{n} Q\right)+\cos \left(k_{n} Q\right)}{k_{n}{ }^{2}}+c_{i} \frac{\left(k_{n}{ }^{2} x^{2}-2\right) \sin \left(k_{n} Q\right)+2 k_{n} Q \cos \left(k_{n} Q\right)}{k_{n}{ }^{3}}+\right. \\
\left.d_{i} \frac{k_{n} Q\left(k_{n}{ }^{2} x^{2}-6\right) \sin \left(k_{n} Q\right)+3\left(k_{n}{ }^{2} x^{2}-2\right) \cos \left(k_{n} Q\right)}{k_{n}{ }^{4}}\right)
\end{gathered}
$$

if $\varphi_{m}=\frac{1}{\sqrt{L}} \sin \left(k_{m} Q\right)$ and $\varphi_{n}=\frac{1}{\sqrt{L}} \sin \left(k_{n} Q\right)$,

$$
\begin{aligned}
& V_{m n, i}= \\
& \frac{1}{L}\left(a_{i} \frac{k_{n} \sin \left(k_{m} \mathrm{Q}\right) \cos \left(k_{n} Q\right)-k_{m} \cos \left(k_{m} \mathrm{Q}\right) \sin \left(k_{n} Q\right)}{k_{m}{ }^{2}-k_{n}{ }^{2}}+\frac{b_{i}}{2}\left(\frac{Q \sin \left(\left(k_{m}-k_{n}\right) \mathrm{Q}\right)}{k_{m}-k_{n}}-\frac{Q \sin \left(\left(k_{m}+k_{n}\right) \mathrm{Q}\right)}{k_{m}+k_{n}}+\frac{\cos \left(\left(k_{m}-k_{n}\right) \mathrm{Q}\right)}{\left(k_{m}-k_{n}\right)^{2}}-\right.\right. \\
& \left.\frac{\cos \left(\left(k_{m}+k_{n}\right) \mathrm{Q}\right)}{\left(k_{m}+k_{n}\right)^{2}}\right)+\frac{c_{i}}{2}\left(\frac{\left(k_{m}^{2} Q^{2}-2 k_{m} k_{n} Q^{2}+k_{n}{ }^{2} Q^{2}-2\right) \sin \left(\left(k_{m}-k_{n}\right) \mathrm{Q}\right)}{\left(k_{m}-k_{n}\right)^{3}}-\frac{\left(k_{m}{ }^{2} Q^{2}+2 k_{m} k_{n} Q^{2}+k_{n}{ }^{2} Q^{2}-2\right) \sin \left(\left(k_{m}+k_{n}\right) \mathrm{Q}\right)}{\left(k_{m}+k_{n}\right)^{3}}+\right. \\
& \left.\frac{2 Q \cos \left(\left(k_{m}-k_{n}\right) \mathrm{Q}\right)}{\left(k_{m}-k_{n}\right)^{2}}-\frac{2 Q \cos \left(\left(k_{m}+k_{n}\right) \mathrm{Q}\right)}{\left(k_{m}+k_{n}\right)^{2}}\right)+ \\
& \frac{d_{i}}{2}\left(\frac{Q\left(k_{m}^{2} Q^{2}-2 k_{m} k_{n} Q^{2}+k_{n}^{2} Q^{2}-6\right) \sin \left(\left(k_{m}-k_{n}\right) \mathrm{Q}\right)}{\left(k_{m}-k_{n}\right)^{3}}-\frac{Q\left(k_{m}^{2} Q^{2}+2 k_{m} k_{n} Q^{2}+k_{n}^{2} Q^{2}-6\right) \sin \left(\left(k_{m}+k_{n}\right) \mathrm{Q}\right)}{\left(k_{m}+k_{n}\right)^{3}}+\right. \\
& \left.\left.\frac{3\left(k_{m}^{2} Q^{2}-2 k_{m} k_{n} Q^{2}+k_{n}^{2} Q^{2}-2\right) \cos \left(\left(k_{m}-k_{n}\right) \mathrm{Q}\right)}{\left(k_{m}-k_{n}\right)^{4}}-\frac{3\left(k_{m}^{2} Q^{2}+2 k_{m} k_{n} Q^{2}+k_{n}^{2} Q^{2}-2\right) \cos \left(\left(k_{m}+k_{n}\right) \mathrm{Q}\right)}{\left(k_{m}+k_{n}\right)^{4}}\right)\right) \text {; }
\end{aligned}
$$

if $\varphi_{m}=\frac{1}{\sqrt{L}} \sin \left(k_{m} Q\right)$ and $\varphi_{n}=\frac{1}{\sqrt{L}} \cos \left(k_{n} Q\right)$,

$$
\begin{aligned}
& V_{m n, i}=\frac{1}{L}\left(-a_{i} \frac{k_{n} \sin \left(k_{m} \mathrm{Q}\right) \sin \left(k_{n} Q\right)+k_{m} \cos \left(k_{m} \mathrm{Q}\right) \cos \left(k_{n} Q\right)}{k_{m}{ }^{2}-k_{n}{ }^{2}}+\frac{b_{i}}{2}\left(-\frac{Q \cos \left(\left(k_{m}-k_{n}\right) \mathrm{Q}\right)}{k_{m}-k_{n}}-\frac{Q \cos \left(\left(k_{m}+k_{n}\right) \mathrm{Q}\right)}{k_{m}+k_{n}}+\right.\right. \\
& \left.\frac{\sin \left(\left(k_{m}-k_{n}\right) \mathrm{Q}\right)}{\left(k_{m}-k_{n}\right)^{2}}+\frac{\sin \left(\left(k_{m}+k_{n}\right) \mathrm{Q}\right)}{\left(k_{m}+k_{n}\right)^{2}}\right)+ \\
& \frac{c_{i}}{2}\left(-\frac{\left(k_{m}^{2} Q^{2}-2 k_{m} k_{n} Q^{2}+k_{n}^{2} Q^{2}-2\right) \cos \left(\left(k_{m}-k_{n}\right) \mathrm{Q}\right)}{\left(k_{m}-k_{n}\right)^{3}}-\frac{\left(k_{m}^{2} Q^{2}+2 k_{m} k_{n} Q^{2}+k_{n}^{2} Q^{2}-2\right) \cos \left(\left(k_{m}+k_{n}\right) \mathrm{Q}\right)}{\left(k_{m}+k_{n}\right)^{3}}+\frac{2 Q \sin \left(\left(k_{m}-k_{n}\right) \mathrm{Q}\right)}{\left(k_{m}-k_{n}\right)^{2}}+\right. \\
& \left.\frac{2 Q \sin \left(\left(k_{m}+k_{n}\right) Q\right)}{\left(k_{m}+k_{n}\right)^{2}}\right)+ \\
& \frac{d_{i}}{2}\left(-\frac{Q\left(k_{m}^{2} Q^{2}-2 k_{m} k_{n} Q^{2}+k_{n}^{2} Q^{2}-6\right) \cos \left(\left(k_{m}-k_{n}\right) \mathrm{Q}\right)}{\left(k_{m}-k_{n}\right)^{3}}-\frac{Q\left(k_{m}{ }^{2} Q^{2}+2 k_{m} k_{n} Q^{2}+k_{n}^{2} Q^{2}-6\right) \cos \left(\left(k_{m}+k_{n}\right) \mathrm{Q}\right)}{\left(k_{m}+k_{n}\right)^{3}}+\right. \\
& \left.\left.\frac{3\left(k_{m}^{2} Q^{2}-2 k_{m} k_{n} Q^{2}+k_{n}^{2} Q^{2}-2\right) \sin \left(\left(k_{m}-k_{n}\right) \mathrm{Q}\right)}{\left(k_{m}-k_{n}\right)^{4}}+\frac{3\left(k_{m}^{2} Q^{2}+2 k_{m} k_{n} Q^{2}+k_{n}^{2} Q^{2}-2\right) \sin \left(\left(k_{m}+k_{n}\right) \mathrm{Q}\right)}{\left(k_{m}+k_{n}\right)^{4}}\right)\right) \text {; }
\end{aligned}
$$

if $\varphi_{m}=\frac{1}{\sqrt{L}} \cos \left(k_{m} Q\right)$ and $\varphi_{n}=\frac{1}{\sqrt{L}} \cos \left(k_{n} Q\right)$,

$$
\begin{gathered}
V_{m n, i}= \\
\frac{1}{L}\left(a_{i} \frac{k_{m} \sin \left(k_{m} \mathrm{Q}\right) \cos \left(k_{n} Q\right)-k_{n} \cos \left(k_{m} \mathrm{Q}\right) \sin \left(k_{n} Q\right)}{k_{m}{ }^{2}-k_{n}{ }^{2}}+\frac{b_{i}}{2}\left(\frac{Q \sin \left(\left(k_{m}-k_{n}\right) \mathrm{Q}\right)}{k_{m}-k_{n}}+\frac{Q \sin \left(\left(k_{m}+k_{n}\right) \mathrm{Q}\right)}{k_{m}+k_{n}}+\frac{\cos \left(\left(k_{m}-k_{n}\right) \mathrm{Q}\right)}{\left(k_{m}-k_{n}\right)^{2}}+\right.\right. \\
\left.\frac{\cos \left(\left(k_{m}+k_{n}\right) \mathrm{Q}\right)}{\left(k_{m}+k_{n}\right)^{2}}\right)+\frac{c_{i}}{2}\left(\frac{\left(k_{m}{ }^{2} Q^{2}-2 k_{m} k_{n} Q^{2}+k_{n}{ }^{2} Q^{2}-2\right) \sin \left(\left(k_{m}-k_{n}\right) \mathrm{Q}\right)}{\left(k_{m}-k_{n}\right)^{3}}+\frac{\left(k_{m}{ }^{2} Q^{2}+2 k_{m} k_{n} Q^{2}+k_{n}{ }^{2} Q^{2}-2\right) \sin \left(\left(k_{m}+k_{n}\right) \mathrm{Q}\right)}{\left(k_{m}+k_{n}\right)^{3}}+\right. \\
\left.\frac{2 Q \cos \left(\left(k_{m}-k_{n}\right) \mathrm{Q}\right)}{\left(k_{m}-k_{n}\right)^{2}}+\frac{2 Q \cos \left(\left(k_{m}+k_{n}\right) \mathrm{Q}\right)}{\left(k_{m}+k_{n}\right)^{2}}\right)+
\end{gathered}
$$




$$
\begin{aligned}
& \frac{d_{i}}{2}\left(\frac{Q\left(k_{m}^{2} Q^{2}-2 k_{m} k_{n} Q^{2}+k_{n}^{2} Q^{2}-6\right) \sin \left(\left(k_{m}-k_{n}\right) \mathrm{Q}\right)}{\left(k_{m}-k_{n}\right)^{3}}+\frac{Q\left(k_{m}{ }^{2} Q^{2}+2 k_{m} k_{n} Q^{2}+k_{n}{ }^{2} Q^{2}-6\right) \sin \left(\left(k_{m}+k_{n}\right) \mathrm{Q}\right)}{\left(k_{m}+k_{n}\right)^{3}}+\right. \\
& \left.\left.\frac{3\left(k_{m}{ }^{2} Q^{2}-2 k_{m} k_{n} Q^{2}+k_{n}{ }^{2} Q^{2}-2\right) \cos \left(\left(k_{m}-k_{n}\right) \mathrm{Q}\right)}{\left(k_{m}-k_{n}\right)^{4}}+\frac{3\left(k_{m}{ }^{2} Q^{2}+2 k_{m} k_{n} Q^{2}+k_{n}{ }^{2} Q^{2}-2\right) \cos \left(\left(k_{m}+k_{n}\right) \mathrm{Q}\right)}{\left(k_{m}+k_{n}\right)^{4}}\right)\right) .
\end{aligned}
$$

For the cases where $m=n$, if $\varphi_{m}=\varphi_{n}=\frac{1}{\sqrt{2 L}}$

$$
V_{m n, i}=\frac{1}{2 L}\left(a_{i} Q+\frac{b_{i}}{2} Q^{2}+\frac{c_{i}}{3} Q^{3}+\frac{d_{i}}{4} Q^{4}\right)
$$

if $\varphi_{m}=\varphi_{n}=\frac{1}{\sqrt{L}} \sin \left(k_{m} Q\right)$

$$
\begin{gathered}
V_{m n, i}= \\
\frac{1}{L}\left(a_{i}\left(\frac{Q}{2}-\frac{\sin \left(2 k_{m} Q\right)}{4 k_{m}}\right)+b_{i}\left(-\frac{2 k_{m} Q\left(\sin \left(2 k_{m} Q\right)-k_{m} Q\right)+\cos \left(2 k_{m} Q\right)}{8 k_{m}^{2}}\right)+\right. \\
c_{i}\left(\frac{4 k_{m}^{3} Q^{3}+\left(3-6 k_{m}^{2} Q^{2}\right) \sin \left(2 k_{m} Q\right)-6 k_{m} Q \cos \left(2 k_{m} Q\right)}{24 k_{m}^{3}}\right)+ \\
\left.d_{i}\left(\frac{2 k_{m}{ }^{4} Q^{4}+\left(6 k_{m} Q-4 k_{m}{ }^{3} Q^{3}\right) \sin \left(2 k_{m} Q\right)+\left(3-6 k_{m}{ }^{2} Q^{2}\right) \cos \left(2 k_{m} Q\right)}{16 k_{m}{ }^{4}}\right)\right)
\end{gathered}
$$

if $\varphi_{m}=\varphi_{n}=\frac{1}{\sqrt{L}} \cos \left(k_{m} Q\right)$,

$$
\begin{gathered}
V_{m n, i}= \\
\frac{1}{L}\left(a_{i}\left(\frac{Q}{2}+\frac{\sin \left(2 k_{m} Q\right)}{4 k_{m}}\right)+b_{i}\left(\frac{2 k_{m} Q\left(\sin \left(2 k_{m} Q\right)+k_{m} Q\right)+\cos \left(2 k_{m} Q\right)}{8 k_{m}^{2}}\right)+\right. \\
c_{i}\left(\frac{4 k_{m}^{3} Q^{3}+\left(6 k_{m}{ }^{2} Q^{2}-3\right) \sin \left(2 k_{m} Q\right)+6 k_{m} Q \cos \left(2 k_{m} Q\right)}{24 k_{m}^{3}}\right)+ \\
\left.d_{i}\left(\frac{2 k_{m}{ }^{4} Q^{4}+\left(4 k_{m}{ }^{3} Q^{3}-6 k_{m} Q\right) \sin \left(2 k_{m} Q\right)+\left(6 k_{m}{ }^{2} Q^{2}-3\right) \cos \left(2 k_{m} Q\right)}{16 k_{m}}\right)\right)
\end{gathered}
$$

if $\varphi_{m}=\frac{1}{\sqrt{L}} \sin \left(k_{m} Q\right)$ and $\varphi_{n}=\frac{1}{\sqrt{L}} \cos \left(k_{m} Q\right)$,

$$
\begin{gathered}
V_{m n, i}=\frac{1}{L}\left(a_{i}\left(-\frac{\cos ^{2}\left(k_{m} \mathrm{Q}\right)}{2 k_{m}}\right)+b_{i}\left(\frac{-2 k_{m} Q \cos \left(2 k_{m} \mathrm{Q}\right)+\sin \left(2 k_{m} Q\right)}{8 k_{m}^{2}}\right)+c_{i}\left(\frac{\left(1-2 k_{m}^{2} Q^{2}\right) \cos \left(2 k_{m} \mathrm{Q}\right)+2 k_{m} Q \sin \left(2 k_{m} \mathrm{Q}\right)}{8 k_{m}^{3}}\right)+\right. \\
\left.d_{i}\left(\frac{\left(6 k_{m} Q-4 k_{m}^{3} Q^{3}\right) \cos \left(2 k_{m} \mathrm{Q}\right)+3\left(2 k_{m}^{2} Q^{2}-1\right) \sin \left(2 k_{m} \mathrm{Q}\right)}{16 k_{m}{ }^{4}}\right)\right)
\end{gathered}
$$


Text S3. Calculation of Thermodynamic Quantities.

The energy and entropy of each internal motion were calculated using the standard expressions:

and

$$
E=N_{A} \frac{\sum_{m}^{M} \epsilon_{m} e^{-\beta \epsilon_{m}}}{q}
$$

$$
S=R \ln (q)+\frac{E}{T}
$$

With ideal gas and rigid rotor assumptions, the global translational and rotational energies and entropies were calculated with

$$
\begin{aligned}
& E_{\text {trans }}=\frac{3}{2} R T \\
& S_{\text {trans }}=R\left[\frac{3}{2} \ln \left(\frac{2 \pi m}{h^{2}}\right)+\frac{5}{2} \ln (k T)-\ln (p)+\frac{5}{2}\right] \\
& E_{\text {rot }}=\frac{3}{2} R T \\
& S_{\text {rot }}=R\left[\ln \left(\frac{8 \pi^{2}}{\sigma}\right)+\frac{3}{2} \ln \left(\frac{2 \pi k T}{h^{2}}\right)+\frac{1}{2} \ln \left(I_{A} I_{B} I_{C}\right)+\frac{3}{2}\right],
\end{aligned}
$$

where $m$ is the molecular mass, $I_{A}, I_{B}$, and $I_{C}$ are the principal moments of inertia of the system; $\sigma$ is the external symmetry number for the system (see Table $\mathrm{S} 1$ for $\sigma \mathrm{s}$ of the systems discussed in this work). The total entropy of a system was calculated by summing the contributions of global translation, rotation, and all the internal motions. The total enthalpy was calculated by summing all these contributions and adding $R T$. 
Table S1. External symmetry number $(\sigma)$ for the molecules considered in this work.

\begin{tabular}{cccc}
\hline & $\sigma(-)$ & & $\sigma(-)$ \\
\hline Ethane & 6 & 1-Butene & 1 \\
Propane & 2 & 1,3-Butadiene & 2 \\
n-Butane & 2 & Ethyl Methyl Ether & 1 \\
Isobutane & 3 & Ethanol & 1 \\
n-Pentane & 2 & Allyl Alcohol & 1 \\
Isopentane & 1 & Propionaldehyde & 1 \\
Neopentane & 12 & 2-Butanone & 1 \\
n-Hexane & 2 & Acetic Acid & 1 \\
2,2-Dimethylbutane & 1 & Propylamine & 1 \\
2,3-Dimethylbutane & 2 & 1-Nitropropane & 1 \\
2-Methylpentane & 1 & Methylnitrite & 1 \\
3-Methylpentane & 1 & 1-Fluoropropane & 1 \\
n-Heptane & 2 & 1-Chloropropane & 1 \\
2,2-Dimethylpentane & 1 & 1-Bromopropane & 1 \\
2,3-Dimethylpentane & 1 & Ethyl Methyl Sulfide & 1 \\
2,4-Dimethylpentane & 2 & Methyl Disulfide & 2 \\
3,3-Dimethylpentane & 2 & Ethanethiol & 1 \\
3-Ethylpentane & 3 & Ethylene Glycol & 2 \\
2-Methylhexane & 1 & Acrylic Acid & 1 \\
3-Methylhexane & 1 & & \\
2,2,3-Trimethylbutane & 1 & & \\
n-Octane & 2 & & \\
\hline
\end{tabular}

